\title{
Probing the Single-Particle Neutron Origin of the Pygmy Dipole Resonance
}

\author{
N. Tsoneva \\ Extreme Light Infrastructure (ELI-NP), Horia Hulubei National Institute for \\ R\&D in Physics and Nuclear Engineering (IFIN-HH), Str. Reactorului No. 30, \\ 077125 Bucharest-Măgurele, Romania
}

Received 22 October 2021

doi: https://doi.org/10.55318/bgjp.2021.48.5-6.587

\begin{abstract}
The first comprehensive study of the single-particle structure of pygmy dipole resonance (PDR) in double-magic ${ }^{208} \mathrm{~Pb}$ and semi-magic ${ }^{120} \mathrm{Sn}$ nuclei with an excess of neutrons is presented. Theoretical calculations are carried out with a microscopic approach based on the energy-density functional plus quasiparticle-phonon model $(\mathrm{EDF}+\mathrm{QPM})$ and combined with nuclear reaction theory. The obtained theoretical results are compared to novel ${ }^{207} \mathrm{~Pb}(\mathrm{~d}, \mathrm{p}){ }^{208} \mathrm{~Pb}$ and ${ }^{119} \mathrm{Sn}(\mathrm{d}, \mathrm{p} \gamma){ }^{120} \mathrm{Sn}$ experiments and other available experimental data. In addition, various properties of the dipole strength distributions are discussed in order to elucidate the microscopic structure of the PDR in detail. The studies will support ELI-Gamma-Above-Neutron-Threshold (ELIGANT) day-one experiments at the ELI-NP.
\end{abstract}

KEY WORDS: pygmy dipole resonance, microscopic nuclear structure and reaction theory, ${ }^{207} \mathrm{~Pb}(\mathrm{~d}, \mathrm{p}){ }^{208} \mathrm{~Pb}$ and ${ }^{119} \mathrm{Sn}(\mathrm{d}, \mathrm{p} \gamma){ }^{120} \mathrm{Sn}$ reactions.

\section{Introduction}

The present research points to new aspects of the nature of PDR, which emerges as a common feature of isospin asymmetric nuclei with excess neutrons that form a neutron skin $[1,2]$. Theoretical studies of low-energy dipole spectral distributions and transition densities in relation to PDR have found that this excitation mode is compatible with neutron skin vibrations against the isospinsymmetric nuclear 'core' [3-6]. In addition, the presence of a neutron skin may influence the nuclear response on external electromagnetic and hadronic fields. An observable which could be sensitive to induced skin effects on nuclear excitations, especially at low-energies, is the dipole polarizability. The latter is proportional to the second moment of the dipole photoabsorption cross section and it might also be useful for constraining the neutron skin and symmetry energy, the nuclear matter equation of state and properties of neutron 
stars [7]. Typically, the PDR appears as an additional dipole strength component below the neutron threshold sitting on top of the low-energy tail of the giant dipole resonance (GDR) which is classically represented by a Lorentzian shape $[1,8]$. Recent studies of nuclear reactions of astrophysical interest show that the reaction cross-sections strongly depend on the low-energy part of the electromagnetic strength function which is connected to the dipole photoabsorption cross section $[7,9,10]$. Such enhancement of low-energy GDR tail can have important effects on neutron and other particle capture reaction rates important for nucleosynthesis of heavier elements in stellar environments [7,9]. However, the PDR is a nuclear quantum effect. The detailed systematic analysis of pygmy dipole strengths in different nuclei shows that standard strength functions based on Lorentz curves, currently widely used for the calculation of nucleon-capture cross sections by statistical reaction models, do not describe dipole spectral distributions below the $(\gamma, \mathrm{n})$ threshold correctly and need to be improved [8]. In this regard, the microscopic structure of the PDR must be explicitly taken into account [6]. Microscopic theoretical models explain the PDR as a sequence of excited one-particle-one-hole $(1 p-1 h)$ excitations acting coherently and, therefore, causing enhanced transition strength [6,11]. However, the PDR degree of collectivity is still in question and it has not been experimentally accessed. Here we present for the first time state-of-the-art theoretical calculations in comparison with novel experimental ${ }^{207} \mathrm{~Pb}(\mathrm{~d}, \mathrm{p})^{208} \mathrm{~Pb}$ and ${ }^{119} \mathrm{Sn}(\mathrm{d}, \mathrm{p} \gamma){ }^{120} \mathrm{Sn}$ reaction data that access theoretical wave functions and can clearly prove the one-particle neutron character of the PDR [12,13]. Another interesting aspect of the low-energy excitations and in particular of the PDR is the role of multi-particle-multi-hole couplings and core polarization effects induced by the GDR $[6,7]$. For this purpose, an extended theoretical approach, which explicitly takes into account the interactions between multi-quasiparticle configurations, based on EDF methods [14] and multiphonon techniques of the QPM [15] is applied. The latter is used for predictions and systematic investigations of nuclear ground and excited states [5,6,16-18]. Recently the EDF+QPM approach was used to describe the fine structure of nuclear electric and magnetic excitations below the neutron threshold, $\mathrm{S}_{n}=8.087 \mathrm{MeV}$, as well as total dipole photoabsorption cross-section and GDR in semi-magic ${ }^{206} \mathrm{~Pb}$ nucleus. Theoretical results show that the PDR increases ${ }^{205} \mathrm{~Pb}(\mathrm{n}, \gamma)^{206} \mathrm{~Pb}$ reaction rate by about an order of magnitude (for more details see in Ref. [7]).

\section{The Theoretical Approach}

The QPM model Hamiltonian [15] is given by:

$$
H=H_{\mathrm{MF}}+H_{\text {res }},
$$

where $H_{\mathrm{MF}}$ is a mean-field part and $H_{\text {res }}$ stands for the residual interaction.

The mean-field (MF) part $H_{M F}$ is treated by self-consistent Skyrme Hartree- 
Fock-Bogoliubov (HFB) theory described in $[6,14]$. The pure HFB picture is in fact extended beyond MF by dynamical self-energies, hence incorporating a more detailed spectral description of nuclear spectra. The procedure allows us to account in a self-consistent manner for nuclear binding energies and other ground-state properties of nuclei like the charge radii and the neutron skin thickness $[4,6,14]$.

The nuclear excited states are calculated with a residual interaction which is based on the QPM formalism [15]

$$
H_{\mathrm{res}}=H_{M}^{p h}+H_{S M}^{p h}+H_{M}^{p p},
$$

where effective interactions are implemented to account for the interaction between the quasiparticles. The terms $H_{M}^{p h}, H_{S M}^{p h}$ and $H_{M}^{p p}$ are taken as a sum of isoscalar and isovector separable multipole and spin-multipole interactions in the particle-hole $(p-h)$ and multipole pairing interaction in the particle-particle $(p-p)$ channels, respectively [15]. The model parameters are fixed empirically in such a way that the properties of the lowest-lying collective states and giant resonances are described accurately [19].

The nuclear excitations are expressed in terms of quasiparticle-random-phaseapproximation (QRPA) phonons,

$$
Q_{\lambda \mu i}^{+}=\frac{1}{2} \sum_{j j^{\prime}}\left(\psi_{j j^{\prime}}^{\lambda i} A_{\lambda \mu}^{+}\left(j j^{\prime}\right)-\varphi_{j j^{\prime}}^{\lambda i} \widetilde{A}_{\lambda \mu}\left(j j^{\prime}\right)\right),
$$

where the set of quantum numbers $j \equiv(n l j m \tau)$ label single-nucleon states, and $A_{\lambda \mu}^{+}$and $\widetilde{A}_{\lambda \mu}$ are the time-forward and time-backward two-quasiparticle (2QP) operators, creating or annihilating two quasiparticles coupled to a total angular momentum $\lambda$ with projection $\mu$ [15]. The excitation energies of the phonons and the time-forward and time-backward amplitudes $\psi_{j_{1} j_{2}}^{\lambda i}$ and $\varphi_{j_{1} j_{2}}^{\lambda i}$ in Eq. (3) are determined by solving QRPA equations [15].

Thus, for spherical even-even nuclei the model Hamiltonian is diagonalized on an orthonormal set of wave functions constructed from one-, two- and threephonon configurations [20]:

$$
\begin{gathered}
\Psi_{\nu}(J M)=\left\{\sum_{i} R_{i}(J \nu) Q_{J M i}^{+}+\sum_{\substack{\lambda_{1} i_{1} \\
\lambda_{2} i_{2}}} P_{\lambda_{2} i_{2}}^{\lambda_{1} i_{1}}(J \nu)\left[Q_{\lambda_{1} \mu_{1} i_{1}}^{+} \times Q_{\lambda_{2} \mu_{2} i_{2}}^{+}\right]_{J M}\right. \\
\left.+\sum_{\substack{\lambda_{1} i_{1} \lambda_{2} i_{2} \\
\lambda_{3} i_{3} I}} T_{\lambda_{3} i_{3}}^{\lambda_{1} i_{1} \lambda_{2} i_{2} I}(J \nu)\left[\left[Q_{\lambda_{1} \mu_{1} i_{1}}^{+} \otimes Q_{\lambda_{2} \mu_{2} i_{2}}^{+}\right]_{I K}^{+} \otimes Q_{\lambda_{3} \mu_{3} i_{3}}^{+}\right]_{J M}\right\} \Psi_{0},
\end{gathered}
$$

where $R, P$ and $T$ are unknown amplitudes, $\nu$ labels the number of the excited states, and $\Psi_{0}$ is the ground state wave function of the even-even nucleus (phonon vacuum). An important advantage of the EDF+QPM approach is the description of the nuclear excitations in terms of QRPA phonons as a building 


\section{N. Tsoneva}

blocks of the three-phonon QPM model space $[15,20]$ which provides a microscopic way to multi-configuration mixing. Thus, the QPM formalism allows for a further expansion of QRPA $p-h$ excitations to multi-particle-multi-hole states in terms of coupling between quasiparticles and phonons [15]. The electromagnetic transition matrix elements are calculated for transition operators including the interaction of quasiparticles and phonons [21] where exact commutation relations are implemented which is a necessary condition in order to satisfy the Pauli principle.

\section{Discussion and Results}

\subsection{Single-particle structure of PDR in double-magic ${ }^{208} \mathrm{~Pb}$}

${ }^{208} \mathrm{~Pb}$ is a heavy double-magic nucleus with excess neutrons that lead to the formation of a neutron skin. It is observed that nuclear dipole excitations, especially at low energies in this nucleus, react sensitively to the presence of a neutron skin and can be related to the vibrations of the neutron skin and the PDR mode.

The first comprehensive investigation of the single-particle character of the PDR in ${ }^{208} \mathrm{~Pb}$ on the basis of new experimental data and their theoretical explanation is discussed in Ref. [12]. Theoretical predictions from EDF+QPM and large-scale-shell-model (LSSM) are compared to novel data which were collected from $(\mathrm{d}, \mathrm{p})$ and resonant proton scattering experiments performed at the Q3D spectrograph of the Maier-Leibnitz Laboratory in Garching, Germany. The $(d, p)$ data were analyzed at three scattering angles. This allows to distinguish the two different transfer configurations through which $1^{-}$states of ${ }^{208} \mathrm{~Pb}$ can be populated from the $J^{\pi}=1 / 2^{-}$ground state of ${ }^{207} \mathrm{~Pb}$; namely $\left(3 p_{1 / 2}\right)^{-1}\left(4 s_{1 / 2}\right)^{+1}(l=0)$, and $\left(3 p_{1 / 2}\right)^{-1}\left(3 d_{3 / 2}\right)^{+1}(l=2)$. The angular distributions are obtained from Distorted-Wave-Born-Approximation (DWBA) model calculations where the global optical-model parameters (OMP) were used for the protons and the deuterons with adjustments to the real potential of the volume Woods-Saxon part [12]. The measured and DWBA angular distributions are found in excellent agreement. In addition, the detailed, high-resolution $(d, p)$ experimental study of the PDR in ${ }^{208} \mathrm{~Pb}$ is supplemented with available experimental data to discuss the microscopic structure of the PDR and its impact on experimental observables by comparing it to the state-the-art theoretical models: $\mathrm{EDF}+\mathrm{QPM}$ and LSSM [12]. The neutron $1 p-1 h$ configurations that contribute to the formation of the PDR are accessed from $(d, p)$ data up to the proton separation energy $S_{p}$ and for a limited number of states from the results of resonant proton scattering via isobaric analog resonances $\left(p, p^{\prime}\right)_{\text {IAR }}$, which probes components that could not be populated in the selective one-neutron transfer reaction. In addition, the experimental observations are compared to the large suite of complementary, experimental data available for ${ }^{208} \mathrm{~Pb}$ and establish $(d, p)$ as an additional, valuable, experimental probe to study the PDR and its collectivity, 


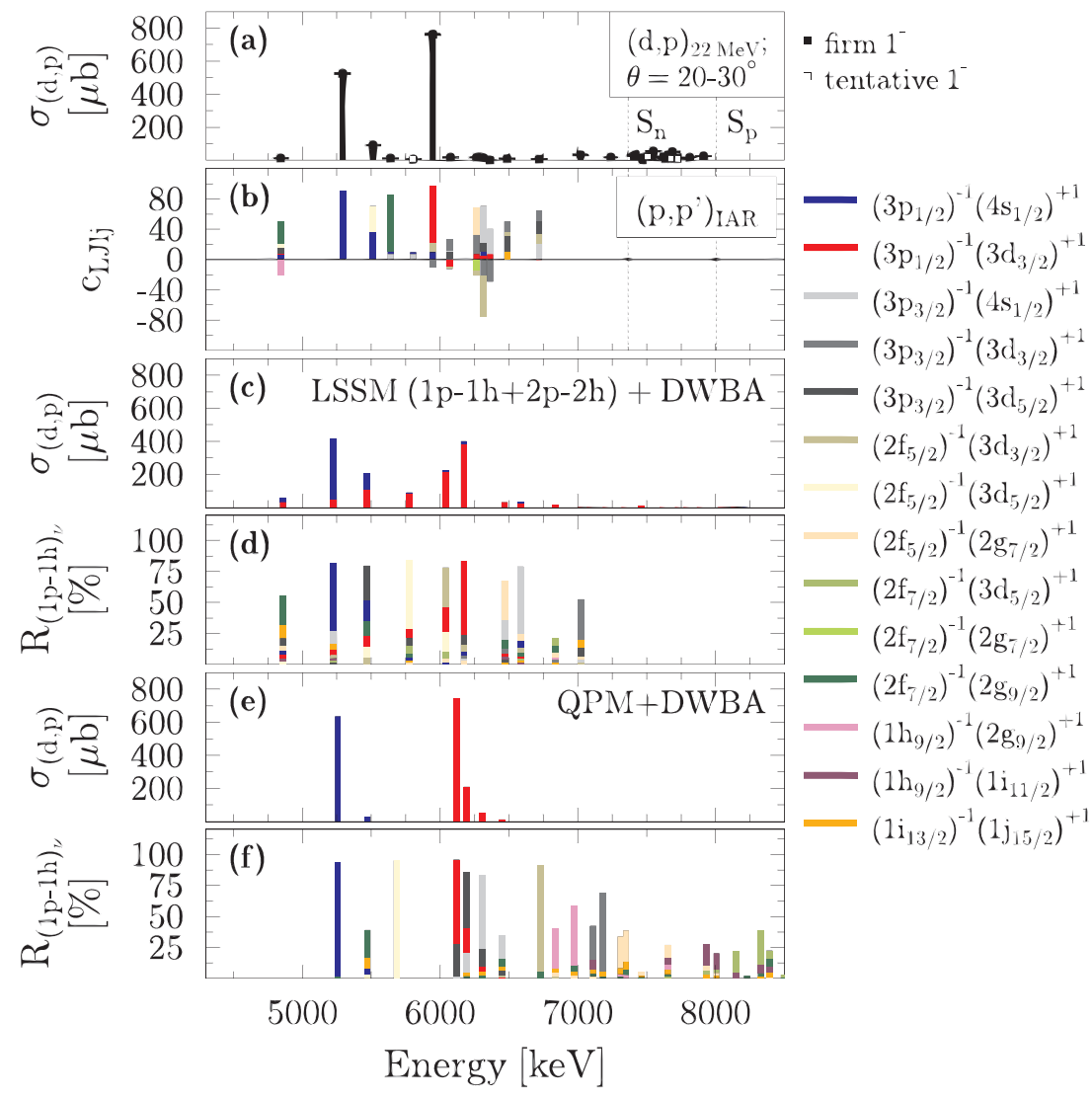

Figure 1. (Color online) (a) Angle-integrated (d,p) cross sections $\sigma(d, p)$; (b) $C_{L J l j}$ amplitudes from $\left(p, p^{\prime}\right)_{\mathrm{IAR}}$; (c) $\sigma(d, p)$ predicted by combining LSSM spectroscopic factors with DWBA calculations; (d) Decomposition of the LSSM wave functions into neutron $1 p-1 h$ components relative to the total wave function $\Psi_{\text {total }}$; (e), (f) same as (c), (d) but for $\mathrm{EDF}+\mathrm{QPM}[12]$.

i.e. the coherence between the different $1 p-1 h$ contributions. The modelindependent, angle-integrated $(d, p)$ cross sections and $C_{L J l j}$ amplitudes from $\left(p, p^{\prime}\right)_{\text {IAR }}$ are shown in Figure $1(\mathrm{a}, \mathrm{b})$ in comparison to a selection of other experimental data on the PDR in ${ }^{208} \mathrm{~Pb}$ [12]. The $(d, p)$ strength pattern shown in Figure 1(a) is dominated by the two strongly populated $1^{-}$states at 5292 and $5947 \mathrm{keV}$, corresponding to the major fragments of the $\left(3 p_{1 / 2}\right)^{-1}\left(4 s_{1 / 2}\right)^{+1}$ $[S=0.77(4)]$ and $\left(3 p_{1 / 2}\right)^{-1}\left(3 d_{3 / 2}\right)^{+1}[S=0.66(4)]$ neutron $1 p-1 h$ strength, respectively. The state spectroscopic factors, $S$, are model dependent but were determined consistently, i.e., using the same OMP as for the angular distributions [12]. Figures 1(c,d,e,f) present the results of EDF+QPM and LSSM cal- 


\section{N. Tsoneva}

culations. The differential cross sections $d \sigma / d \Omega$, predicted theoretical spectroscopic factors, i.e., the overlap of the ${ }^{207} \mathrm{~Pb}$ ground state with excited $1^{-}$ states in ${ }^{208} \mathrm{~Pb}$ when adding a neutron, were combined with the DWBA calculations. Unprecedented access to the theoretical wave functions demonstrating the $1 p-1 h$ neutron origin of the PDR in ${ }^{208} \mathrm{~Pb}$ has been achieved. The comparison of EDF + QPM and LSSM calculations shows that both models were able to account the main features of the $(\mathrm{d}, \mathrm{p})$ data. In particular, EDF+QPM predicts two dominant $\left(3 p_{1 / 2}\right)^{-1}\left(4 s_{1 / 2}\right)^{+1}\left[S_{5.32 \mathrm{MeV}}=0.92\right]$ and $\left(3 p_{1 / 2}\right)^{-1}\left(3 d_{3 / 2}\right)^{+1}$ $\left[S_{6.12 \mathrm{MeV}}=0.68\right]$ fragments in excellent agreement with experiment [12]. For the total (d,p) cross section, the obtained EDF+QPM value is $\Sigma \sigma_{(d, p)(\mathrm{QPM})}=$ $1676 \mu \mathrm{b}$ which is also in very good agreement with experimental data of total $\Sigma \sigma_{(d, p)(\text { (exp.) }}$ which can be taken as a sum of $\sum_{\leq S_{n}} \sigma_{(d, p)(\text { (exp.) }}=1524(17) \mu \mathrm{b}$ below and $\sum_{>S_{n}} \sigma_{(d, p)(\text { exp.) }}=254(9) \mu \mathrm{b}$ above the neutron threshold $S_{n}$, respectively. However, also the QPM does not fragment the $l=0$ and $l=2$ strength sufficiently to describe the strength above $S_{n}$. Due to the doubly-magic nature of ${ }^{208} \mathrm{~Pb}$, the $1 p-1 h$ structure of the QRPA phonons dominates the configuration mixing and polarization contributions (compare [7]). In order to improve the fragmentation above the neutron threshold and the comparison with the experiment, dynamic effects beyond the static mean-field could be important.

\subsection{Single-particle structure of PDR in semi-magic ${ }^{120} \mathrm{Sn}$}

In this section, we report on EDF+QPM calculations of single-particle structure of the PDR in semi-magic nucleus ${ }^{120} \mathrm{Sn}$. In contrast to the case of the doublemagic ${ }^{208} \mathrm{~Pb}$ nucleus, the PDR in an open-shell nucleus ${ }^{120} \mathrm{Sn}$ is characterized by a much higher level density. In comparison, the ${ }^{119} \mathrm{Sn}(d, p \gamma){ }^{120} \mathrm{Sn}$ reaction is studied in a novel experiment performed at the University of Cologne with the combined SONIC@HORUS setup for coincident particle-spectroscopy [13]. Theoretically, a novel approach was developed that combines the EDF + QPM theory with the nuclear reaction model (EDF + QPM + reaction) to consistently derive $(d, p)$ cross sections, $\gamma$-decay branchings, $(d, p \gamma)$ yields, and energyintegrated $\left(\gamma, \gamma^{\prime}\right)$ cross-sections [13].

Since ground-state correlations are small, we describe the ${ }^{119} \mathrm{Sn}$ target as a pure $s_{1 / 2}$ hole in the ${ }^{120} \mathrm{Sn}$ 'core'. Within this approximation, the $(d, p)$ reaction populates one-phonon $1^{-}$states that contain $3 p_{1 / 2}$ and $3 p_{3 / 2}$ one-quasiparticle states, and the corresponding angular differential cross section populating a $1^{-}$ state $\nu$ can be written as

$$
\begin{aligned}
\frac{d \sigma_{\nu}}{d \Omega}(\theta)=\frac{\mu_{i} \mu_{f}}{\left(2 \pi \hbar^{2}\right)^{2}} \frac{k_{f}}{k_{i}} \mid u_{3 p_{1 / 2}} R_{3 p_{1 / 2}}(\nu) \psi_{\frac{1}{2} \frac{1}{2}}^{3 p_{1 / 2}} T_{p_{1 / 2}}(\theta) \\
+\left.u_{3 p_{3 / 2}} R_{3 p_{3 / 2}}(\nu) \psi_{\frac{1}{2} \frac{3}{2}}^{3 p_{3 / 2}} T_{p_{3 / 2}}(\theta)\right|^{2},
\end{aligned}
$$

where $R$ are the one-phonon amplitudes defined with eq. (4), $T_{p_{1 / 2}}$ and $T_{p_{3 / 2}}$ 


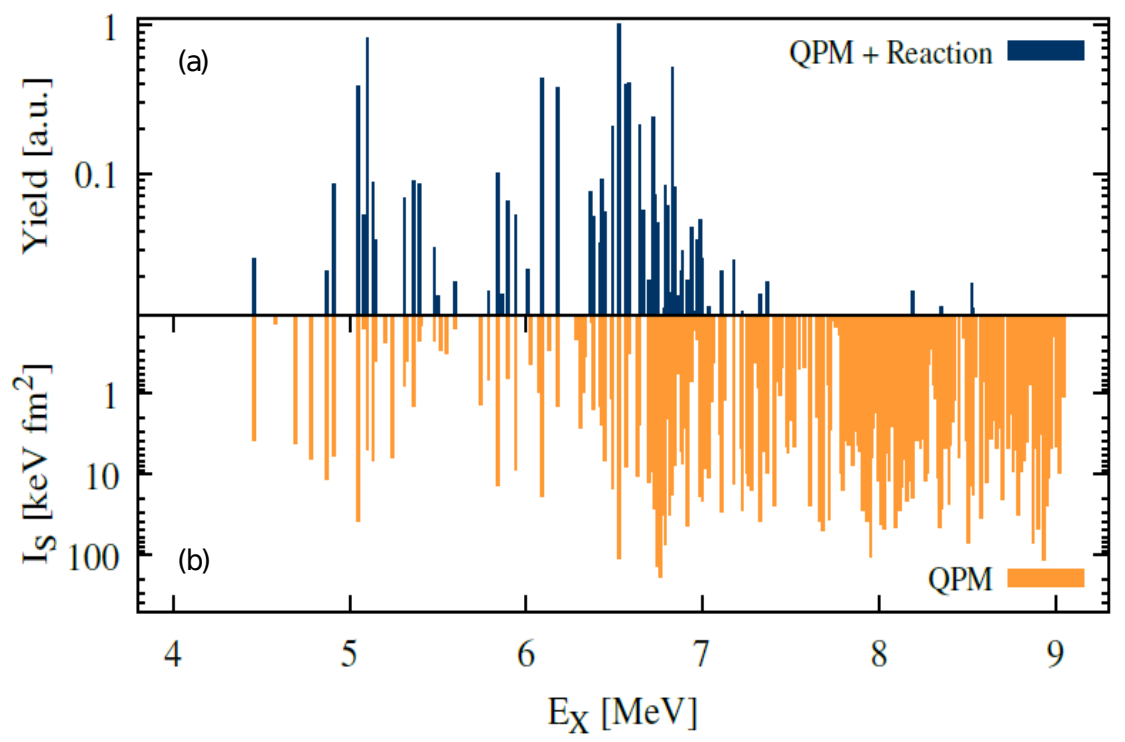

Figure 2. (Color online) (a) relative ${ }^{119} \mathrm{Sn}(d, p \gamma){ }^{120} \mathrm{Sn}$ yields from the $\mathrm{EDF}+\mathrm{QPM}+$ Reaction formalism and (b) predicted energy-integrated cross-sections $I_{S}$, both taking into account $\gamma$-decay branching predicted by the EDF+QPM. Theoretical $(d, p \gamma)$ cross sections were calculated at scattering angles identical to the experiment [13].

are the DWBA $(d, p) T$-matrices (see, e.g. [13]) populating the corresponding $p_{1 / 2}$ and $p_{3 / 2}$ states, respectively, $\theta$ is the deflection angle, $\mu_{i}, \mu_{f}$ are the reduced masses in the incident and outgoing channels, $k_{i}, k_{f}$ are the respective momenta, $u_{3 p_{3 / 2}}$ and $u_{3 p_{1 / 2}}$ are the one-quasiparticle occupation numbers obtained by solving BCS equations [15]. In order to describe the observed fragmentation pattern of the PDR mode in semi-magic nucleus ${ }^{120} \mathrm{Sn}$, one must go beyond QRPA and to take into account the interaction between quasiparticles and phonons. That is achieved by the three-phonon QPM approach, introduced in Section 2. Since ${ }^{120} \mathrm{Sn}$, in contrast to ${ }^{208} \mathrm{~Pb}$, is an open-neutron-shell nucleus $(N=70)$, two- and three-phonon confgurations already contribute at lower energies (compare Figure 1 and Figure 2). Below $7 \mathrm{MeV}$, one-phonon confgurations dominate the picture though. Above $7 \mathrm{MeV}$, two-phonon and three-phonon contributions begin to contribute signifcantly to the spectral distribution. The theoretical results of $(d, p \gamma)$ yields, and energy-integrated $\left(\gamma, \gamma^{\prime}\right)$ cross-sections in ${ }^{120} \mathrm{Sn}$ are shown in Figure 2 and they are found in very good agreement with the novel (d,p $\gamma)$ experiment from Ref. [13]. The theoretical $(d, p \gamma)$ strength is fragmented at lower energies and it reproduces the experimental centroid energy of $6.49 \mathrm{MeV}$ as well as summed $\left(\gamma, \gamma^{\prime}\right)$ cross-sections for states excited with both probes. 
In addition, we calculated the $\gamma$-decay branching ratios of the ground-state $b_{0}$ and excited $2^{+}$states, $b_{i}$ in order to examine in detail the low-energy dipole strength associated with the PDR in ${ }^{120} \mathrm{Sn}$ [12]. Since the observation of large values of $b_{0}$ is a signature of the most important one-phonon contribution to excited $1^{-}$states [18], the observation of large $b_{i}$ values is a clear indication of multi-phonon component in these states. The obtained results emphasize that the branching ratios serve as a highly sensitive observable for distinguishing between single $p-h$ configurations and multiphonon structures $[13,18]$. The analysis shows that EDF + QPM reproduces the proposed experimental decrease in the ground state $\gamma$-decay branching ratios towards higher excitation energies due to more complex configurations.

\section{Conclusions}

The new findings from the advanced EDF + QPM and LSSM theories and novel experimental $(d, p)$ and $(d, p \gamma)$ data have proven the neutron single-particle origin of PDR in double-magic ${ }^{208} \mathrm{~Pb}$ and semi-magical ${ }^{120} \mathrm{Sn}$ nuclei. In addition they establish $(d, p)$ and $(d, p \gamma)$ reactions as an additional, valuable, experimental probe for studying microscopic structure PDR and its collectivity. This is achieved through unprecedented access to theoretical wave functions that demonstrate the $1 p-1 h$ neutron origin of PDR in these nuclei. Furthermore, it turns out that the knowledge of the microscopic structure PDR is of crucial importance for the precise determination of $(n, \gamma)$ reaction rates of key elements of s- and r-process of stellar nucleosynthesis. Even more, when approaching the limits of existence of atomic nuclei which are characterized by a very short life time, reliable microscopic calculations become of prime interest for such astrophysics applications.

With the unique possibilities of the VEGA system, the ELI-NP facility can deliver high-resolution data that originate from photon-induced excitations and reactions. Particular attention will be paid to the GDR and the PDR ground state $\gamma$-decays, as well as investigations of multi-level $\gamma$-decays through low-lying states which enable new insights into the dynamics of isospin-asymmetric nuclei, nuclear matter and the properties of neutron stars.

\section{Acknowledgements}

This work was partly supported by EU Development Fund and Competitiveness Operational Program for the Extreme Light Infrastructure -Nuclear Physics (ELI-NP) Project Phase II (1/07.07.2016, COP, ID1334) and the contract PN 19 060105 sponsored by the Romanian Ministry of Research and Innovation. 
Probing the Single-Particle Neutron Origin of the Pygmy Dipole Resonance

\section{References}

[1] D. Savran, T. Aumann, A. Zilges (2013) Experimental studies of the Pygmy Dipole Resonance. Prog. Part. Nucl. Phys. 70 210-245 DOI: https://doi.org/10.1016/ j.ppnp.2013.02.003.

[2] A. Bracco, F.C.L. Crespi, E.G. Lanza (2015) Gamma decay of pygmy states from inelastic scattering of ions. Eur. Phys. J. A 5199 DOI: https://doi.org/10.1140/epja/ i2015-15099-6.

[3] N. Tsoneva, H. Lenske, Ch. Stoyanov (2004) Probing the nuclear neutron skin by low-energy dipole modes. Phys. Lett. B 586 213-218 DOI: 10.1016/j.physletb.2004.02.024.

[4] N. Tsoneva, H. Lenske (2008) Pygmy dipole resonances in the tin region. Phys. Rev. $C 77$ pages $=$ 024321-024337 DOI: https://doi.org/10.1103/PhysRevC.77.024321.

[5] N. Tsoneva, H. Lenske (2011) Pygmy Quadrupole Resonance in Skin Nuclei. Phys. Lett. B 695 174-180 DOI: https://doi.org/10.1016/j.physletb.2010.11.002.

[6] N. Tsoneva and H. Lenske (2016) Energy-Density Functional Plus QuasiparticlePhonon Model Theory as a Powerful Tool for Nuclear Structure and Astrophysics. Physics of Atomic Nuclei 79 885-903 DOI: 10.1134/S1063778816060247.

[7] A.P. Tonchev, N. Tsoneva, C. Bhatia, C.W. Arnold, S. Goriely, S.L. Hammond, J.H. Kelley, E. Kwan, H. Lenske, J. Piekarewicz, R. Raut, G. Rusev, T. Shizuma, W. Tornow (2017) Pygmy and core polarization dipole modes in ${ }^{206} \mathrm{~Pb}$ : Connecting nuclear structure to stellar nucleosynthesis.. Phys. Lett. B 773 20-25 DOI: https://doi.org/10.1016/j.physletb.2017.07.062.

[8] R. Schwengner, R. Massarczyk, G. Rusev, N. Tsoneva, D. Bemmerer, R. Beyer, R. Hannaske, A.R. Junghans, J.H. Kelley, E. Kwan, H. Lenske, M. Marta, R. Raut, K.D. Schilling, A. Tonchev, W. Tornow, and A. Wagner (2013) Phys. Rev. C, 87 024306-024318 DOI: https://doi.org/10.1103/PhysRevC.87.024306.

[9] N. Tsoneva, S. Goriely, H. Lenske, R. Schwengner (2015) Pygmy resonances and radiative nucleon captures for stellar nucleosynthesis. Phys. Rev. C 91044318 044329 DOI: https://doi.org/10.1103/PhysRevC.91.044318.

[10] R. Raut, A.P. Tonchev, G. Rusev, W. Tornow, C. Iliadis, M. Lugaro, J. Buntain, S. Goriely, J.H. Kelley, R. Schwengner, A. Banu, N. Tsoneva (2013) CrossSection Measurements of the ${ }^{86} \operatorname{Kr}(\gamma, \mathrm{n})$ Reaction to Probe the s-Process Branching at ${ }^{85}$ Kr. Phys. Rev. Lett. 111 112501-112506 DOI: https://doi.org/10.1103/ PhysRevLett.111.112501.

[11] A.P. Tonchev, S.L. Hammond, J.H. Kelley, E. Kwan, H. Lenske, G. Rusev, W. Tornow, N. Tsoneva (2010) Spectral Structure of the Pygmy Dipole Resonance. Phys. Rev. Lett. 104 072501-072505 DOI: https://doi.org/10.1103/Phys RevLett.104.072501.

[12] M. Spieker, A. Heusler, B.A. Brown, T. Faestermann, R. Hertenberger, G. Potel, M. Scheck, N. Tsoneva, M. Weinert, H.-F. Wirth, and A. Zilges (2020) Accessing the single-particle structure of the Pygmy Dipole Resonance in ${ }^{208} \mathrm{~Pb}$ Phys.Rev.Lett. 125 102503 DOI: 10.1103/PhysRevLett.125.102503

[13] M. Weinert, M. Spieker, G. Potel, N. Tsoneva, M. Müscher, J. Wilhelmy, and A. Zilges (2021) The microscopic structure of the low-energy electric dipole response of ${ }^{120} \mathrm{Sn}$ Phys. Rev. Lett. accepted. 


\section{N. Tsoneva}

[14] F. Hofmann, H. Lenske Hartree-Fock calculations in the density matrix expansion approach. (1998) Phys. Rev. C, 57 2281-2293 DOI: https://doi.org/10.1103/Phys RevC.57.2281.

[15] V.G. Soloviev (1976) "Theory of complex nuclei" Oxford: Pergamon Press.

[16] L. Pellegri, A. Bracco, N. Tsoneva, R. Avigo, G. Benzoni, N. Blasi, S. Bottoni, F. Camera, S. Ceruti, F.C.L. Crespi, A. Giaz, S. Leoni, H. Lenske, B. Million, A.I. Morales, R. Nicolini, O. Wieland, D. Bazzacco, P. Bednarczyk, B. Birkenbach, M. Ciema la, G. de Angelis, E. Farnea, A. Gadea, A. Görgen, A. Gottardo, J. Grebosz, R. Isocrate, M. Kmiecik, M. Krzysiek, S. Lunardi, A. Maj, K. Mazurek, D. Mengoni, C. Michelagnoli, D.R. Napoli, F. Recchia, B. Siebeck, S. Siem, C. Ur, J.J. Valiente-Dobön (2015) A multitude of $2^{+}$discrete states in ${ }^{124} \mathrm{Sn}$ via the $\left({ }^{17} \mathrm{O},{ }^{17} \mathrm{O}^{\prime} \gamma\right)$ reaction: pygmy quadrupole states. Phys. Rev. C 92 014330-1 014330-6 DOI: https://doi.org/10.1103/PhysRevC.92.014330.

[17] M. Spieker, N. Tsoneva, V. Derya, J. Endres, D. Savran, P. Butler, M.N. Harakeh, S. Harissopulos, R.-D. Herzberg, R. Krücken, A. Lagoyannis, H. Lenske, N. Pietralla, L. Popescu, M. Scheck, F. Schlüter, K. Sonnabend, V.I. Stoica, H.J. Wörtche, A. Zilges (2016) The Pygmy Quadrupole Resonance and NeutronSkin Modes in ${ }^{124}$ Sn. Phys. Lett. B 752 102-107 DOI: http://dx.doi.org/10.1016/ j.physletb.2015.11.004.

[18] N. Tsoneva, M. Spieker, H. Lenske, A. Zilges (2019) Fine structure of the pygmy quadrupole resonance in ${ }^{112,114} \mathrm{Sn}$ isotopes. Nucl. Phys. A 990 183-198 DOI: https://doi.org/10.1016/j.nuclphysa.2019.07.008.

[19] A.I. Vdovin, V.G. Soloviev (1983) Sov. J. Part. Nucl. 14237.

[20] M. Grinberg, Ch. Stoyanov (1994) Distribution of two-phonon strength in even $\mathrm{N}=82$ nuclei Nucl. Phys. A 573 231-244 DOI: https://doi.org/10.1016/03759474(94)90169-4.

[21] V.Yu. Ponomarev, Ch. Stoyanov, N. Tsoneva, M. Grinberg (1998) Boson forbidden low-energy E1-transitions in spherical nuclei. Nucl. Phys. A 635 470-483 DOI: https://doi.org/10.1016/S0375-9474(98)00187-0. 